\title{
Biology of Bone: The Vasculature of the Skeletal System
}

\author{
Emma C. Watson and Ralf H. Adams \\ Max Planck Institute for Molecular Biomedicine, Department of Tissue Morphogenesis, and University \\ of Münster, Faculty of Medicine, D-48149 Münster, Germany \\ Correspondence: ralf.adams@mpi-muenster.mpg.de
}

\begin{abstract}
Blood vessels are essential for the distribution of oxygen, nutrients, and immune cells, as well as the removal of waste products. In addition to this conventional role as a versatile conduit system, the endothelial cells forming the innermost layer of the vessel wall also possess important signaling capabilities and can control growth, patterning, homeostasis, and regeneration of the surrounding organ. In the skeletal system, blood vessels regulate developmental and regenerative bone formation as well as hematopoiesis by providing vascular niches for hematopoietic stem cells. Here we provide an overview of blood vessel architecture, growth and properties in the healthy, aging, and diseased skeletal system.
\end{abstract}

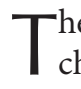
he blood vessel network consists of a hierarchical assembly of endothelial cell (EC)-lined tubes that vary in identity and caliber (Potente and Makinen 2017). To enable the efficient transport of cargo such as gases, nutrients, metabolic products, hormones, and immune cells around the body, ECs form a semipermeable barrier that controls the movement of small and large molecules and actively regulates the transmigration of immune cells between vessels and tissues (Sumpio et al. 2002). Recent studies have also identified a role for ECs in regulating tissue morphogenesis through the secretion of growth factors and cytokines, as well as expression of signaling molecules on the cell surface (Ding et al. 2010, 2011, 2014; Hu et al. 2014; Ramasamy et al. 2015). An intimate link between blood vessel growth, known as angiogenesis, and the formation of new bone (termed osteogenesis) has been demonstrated in numerous studies (Gerber et al. 1999; Maes et al. 2010; Maes 2013).
This chapter will discuss the role blood vessels play in the formation of bone by first introducing the processes of angiogenesis, followed by the process of bone growth and vascularization. Here, we will also discuss the unique architecture of the vasculature in bone as well as the role of the vasculature in a range of bone pathologies.

After the formation of the yolk sac vasculature in the early embryo, the dorsal aorta and the cardinal vein form via a process termed vasculogenesis (Risau and Flamme 1995; Coultas et al. 2005), which involves de novo assembly of mesoderm-derived ECs (or immature EC progenitors). Much or all of the later vascular growth is mediated by the expansion of existing vessel network. This complex process, termed angiogenesis, involves the coordinated proliferation, migration, and differentiation of ECs, which are closely followed by a series of remodeling, specialization, and maturation steps (Potente et al. 2011). While arteries and veins are frequently

Editors: Gerard Karsenty and David T. Scadden

Additional Perspectives on Bone: A Regulator of Physiology available at www.perspectivesinmedicine.org

Copyright (C) 2018 Cold Spring Harbor Laboratory Press; all rights reserved; doi: 10.1101/cshperspect.a031559

Cite this article as Cold Spring Harb Perspect Med 2018;8:a031559 
absent in the most immature vessel networks, the formation and subsequent expansion of these vessels is critical for the establishment of a sufficiently perfused and fully functional vascular bed (Fish and Wythe 2015). In turn, blood flow is also a very critical regulator of EC behavior and gene expression, which contributes to morphological and functional changes in the growing vasculature (Galie et al. 2014; Franco et al. 2016). Accordingly, newly formed and therefore immature blood vessel networks have very different properties compared to those of the adult organism, which may also explain the great difficulty that is associated with the generation of artificial vascular structures in tissue engineering. Furthermore, vessels of different tissues show distinctive characteristics and functional properties. For example, glomerular vessels of the kidney are highly fenestrated (Kamba et al. 2006) to permit efficient blood filtering, while the capillaries of the brain generally display a strong barrier function that protects the brain against the entry of potentially harmful substances and cells from the circulation (Bennett et al. 1959). The same also applies to the vasculature of the skeletal system, which is not only specialized in an organ-specific fashion, but also, as will be discussed further below, shows distinct regional differences in capillary morphology and function.

\section{TIME COURSE OF BONE VASCULARIZATION}

The vasculature of the bone is predominantly, if not exclusively, formed through angiogenesis. Bone formation and vascularization occur concomitantly and can proceed via one of two pathways: endochondral ossification or intramembranous ossification. Endochondral ossification generates the vast majority of bones, including long bones such as the femur and tibia. Long bones typically consist of a long central shaft known as the diaphysis that is capped by the growth plate-containing metaphysis and the distal epiphysis, which is covered by articular cartilage on the outside. In flat bones such as those of the skull, the formation of new bone involves the direct differentiation of mesenchymal cells into osteoblasts in a process termed intramembranous ossification. Flat bones con- sist of a layer of compact bone, interspersed with bone marrow (BM).

Endochondral ossification commences around embryonic day (E)12 in the mouse (Maes et al. 2010). Mesenchymal progenitors aggregate into dense clusters and differentiate into chondrocytes to form cartilage by around E13. The cartilage acts as a scaffold for the future skeleton. At approximately E15, nonproliferative chondrocytes at the center of the cartilage template become hypertrophic and secrete proangiogenic factors such as vascular endothelial growth factor A (VEGF-A). This stimulates blood vessels to invade, along with osteoclasts and osteoprogenitors, forming the primary ossification center (POC). Shortly after birth, at around postnatal day $(\mathrm{P}) 1$, the vasculature extends toward the epiphysis at either end of the bone. As the vasculature expands, the cartilage scaffold is replaced with trabecular bone, facilitating longitudinal bone growth. By P6, the diaphysis and metaphysis are vascularized and display distinct vessel morphologies (Langen et al. 2017). A secondary ossification center (SOC) is established in the epiphysis, leaving a layer of cartilage between the ossification centers, termed the growth plate. In mice, this growth plate persists after puberty, although longitudinal bone growth is substantially diminished. In humans, cartilage deposition ceases at puberty, and the metaphysis and epiphysis subsequently fuse and growth stops (Dirckx et al. 2013).

In contrast to endochondral ossification, intramembranous ossification is more direct, with no cartilage intermediate. It commences with the condensation of mesenchymal cells to form a sponge-like structure. These cells secrete factors such as VEGF-A and extracellular matrix (ECM), which promote the differentiation of mesenchymal cells into osteoprogenitors and osteoblasts to form ossification centers. Blood vessels are attracted to the ossification centers, which they invade, promoting osteogenesis (Percival and Richtsmeier 2013).

\section{BLOOD VESSELS OF BONE}

Bones are highly vascularized and receive around $10 \%-15 \%$ of resting cardiac output 
(Tomlinson and Silva 2013). The structure and function of blood vessels within the bone have long been of interest and early studies used injections of dyes and microradiographs to visualize the vessels within bones (Trueta and Morgan 1960; Zamboni and Pease 1961). This revealed much about the organization of the vasculature of human and animal bones. Bones generally consist of a hard calcified exterior (known as cortical or compact bone) and an inner core of trabecular or cancellous bone containing marrow with hematopoietic stem and progenitor cells. The vasculature of the BM has been shown to influence the formation and maintenance of BM niches essential for hematopoietic stem and progenitor cells and to permit productive hematopoiesis (Mendez-Ferrer et al. 2010; Ding and Morrison 2013; Itkin et al. 2016). A large body of published literature has investigated the subject of vascular stem-cell niches in the BM, so that this interesting but complex topic cannot be addressed here. We therefore refer the reader to some excellent specialized reviews (Morrison and Scadden 2014; Mendez-Ferrer et al. 2015; Asada et al. 2017).

Long bones receive blood supply from multiple sources, including the central nutrient artery, the metaphyseal-epiphyseal arteries, which enter long bones near their distal ends, and the periosteal arteries. Blood flows from the arteries through a dense vessel capillary network and is drained via a large central vein (Trueta 1974). In flat bones, the thickness of the bone was shown to strongly influence the patterning of the microvasculature. Regions thinner than $0.4 \mathrm{~mm}$ possessed only periosteal and dural networks, with larger vessels connecting the two sides of bone in the absence of a true vascular network (Pannarale et al. 1997). On the other hand, thicker flat bones had a microvascular network more similar to that of the long bones. In these regions, distinct periosteal, cortical, and BM networks could be observed (Pannarale et al. 1997).

\section{ENDOTHELIAL CELL SUBTYPES IN BONE}

It has long been noted that blood vessels of different regions in bone have distinct structures, but, until recently, detailed study of the molecular identities of ECs within the bone was limited. In-depth analysis of bone vasculature has revealed a number of EC subtypes with unique molecular identities and specialized functional roles. These distinct capillary EC subpopulations can be identified within the bone based on marker expression and functional characteristics (Kusumbe et al. 2014). Expression levels of cell-surface markers endomucin (Emcn) and CD31 were initially used to distinguish two EC subtypes: type $\mathrm{H}$, a population expressing high levels of the cell-adhesion molecule CD31/ Pecam1 and of the sialoglycoprotein Emcn $\left(\mathrm{CD} 31^{\text {hi }} \mathrm{Emcn}^{\text {hi }}\right)$, and type L, which shows lower expression of these markers $\left(\mathrm{CD} 31^{\mathrm{lo}} \mathrm{Emcn}^{\mathrm{lo}}\right.$ ) (Fig. 1) (Kusumbe et al. 2014). Arterial ECs (AECs) lack Emcn expression after the earliest stages of embryonic development (Brachtendorf et al. 2001), which, together with strong arterial CD31 immunostaining, also facilitates the identification of arteries in relation to other vessel types within the skeletal system. More recently, a third EC subpopulation was identified, type E ECs, so-named because of its abundance in long bones during embryonic and early postnatal development (Langen et al. 2017). Similar to type H ECs, type E ECs are also CD $31^{\text {hi }}$ Emcn $^{\text {hi }}$, but show lower expression of Emcn and higher expression of CD31 relative to type H ECs (Langen et al. 2017). The proportion of each of the EC subpopulations within the bone varies greatly with age. In embryonic development at E16.5, the majority of ECs within the bone are type E, with some type L and only a small number of type H ECs. Following birth, the proportion of type E ECs decreases, accounting for only $2.2 \%$ of bone ECs by P28. The proportion of type L ECs steadily increases throughout life, while the proportion of type $\mathrm{H}$ reaches its maximum at $\mathrm{P} 6$ and declines in adult and aged animals (Langen et al. 2017).

Individual capillary populations of the metaphysis and diaphysis can be distinguished morphologically from around P6 onward (Langen et al. 2017). Different EC subpopulations localize to distinct regions of the bone vasculature. Type H ECs are primarily found in the metaphysis as well as in the endosteum (a thin 


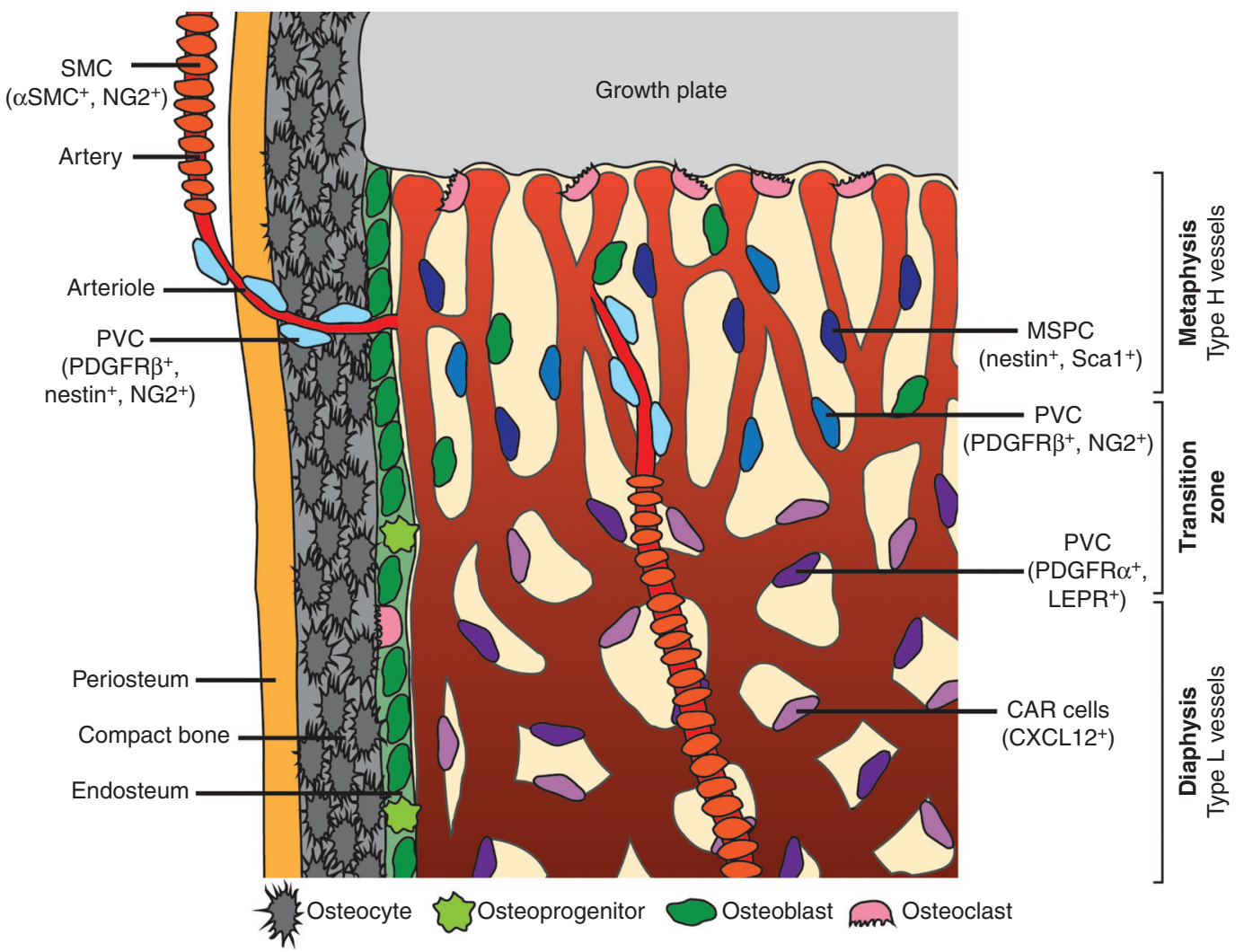

Figure 1. Overview of bone vasculature and perivascular cells (PVCs). Distinct PVC populations are found associated with particular vessel types within the bone. Arteries are associated with smooth muscle cells (SMCs) that express $\alpha$-smooth muscle actin $(\alpha \mathrm{SMA})$ and NG2. Arterioles, which connect arteries to the type $\mathrm{H}$ vasculature of the metaphysis, have associated PVCs that express platelet-derived growth factor receptor (PDGFR) $\beta$, nestin, and NG2. The type $\mathrm{H}$ vessels of the metaphysis are associated with PVCs that express PDGFR $\beta$ and NG2. In this region, mesenchymal stem and progenitor cells (MSPCs) expressing nestin and Scal are also present. Type L sinusoids of the diaphysis associate with PVCs expressing PDGFR $\alpha$ and leptin receptor (LEPR) as well as CXCL12-abundant reticular (CAR) cells.

layer of connective tissue lining the inner surface of compact bone), while type L ECs make up the sinusoidal vasculature of the diaphysis $(\mathrm{Ku}-$ sumbe et al. 2014). Type $H$ vessels of the metaphysis have a distinctive columnar structure. An in-depth study of these vessels revealed that the columns were interconnected via loop-like arches at the distal edge, with bud-shaped protrusions extending toward the hypertrophic growth plate chondrocytes (Fig. 1) (Ramasamy et al. 2016). The bud structures invade the avascular regions of the growth plate where chondrocytes are undergoing apoptosis. The buds anastomose to form the characteristic arch structures of the metaphysis and extend new buds to generate further arches (Ramasamy et al. 2016).

Arteries and distal arterioles do not directly supply the type L sinusoids in long bone. Instead, blood flows from the arteries and arterioles to the type $\mathrm{H}$ vessels of the metaphysis and endosteum. It then passes to the type L sinusoid network at the interface of the metaphysis and diaphysis and is drained from the bone via the large central vein (Ramasamy et al. 2016). In addition, the relative small caliber of arteries and arterioles in bone together with the much larger diameter of postarterial capillaries (Fig. 1) leads to a rapid reduction of blood velocity and 
The Vasculature of the Skeletal System

thereby perfusion within the bone vasculature (Ramasamy et al. 2016; Bixel et al. 2017). These differences in local perfusion and the distinctive arrangement of bone arteries create regions with unique metabolic characteristics and differing oxygen tension. As the diaphysis has no direct arterial blood supply, it remains hypoxic even in postnatal mice, in contrast to the metaphysis (Kusumbe et al. 2014; Ramasamy et al. 2014). Interestingly, direct measurement of local oxygen tension by two-photon phosphorescence lifetime microscopy in the living mouse also showed that the endosteal region is less hypoxic than the deeper perisinusoidal regions of the BM cavity (Spencer et al. 2014).

Furthermore, distinct regions of vasculature can also be distinguished by the presence of differing perivascular support cells (Fig. 1). Perivascular cells associated with arterioles in the metaphysis that connect to type $\mathrm{H}$ capillaries express nestin, platelet-derived growth factor receptor (PDGFR) $\beta$ and NG2 on their cell surface (Kunisaki et al. 2013; Kusumbe et al. 2016; Ramasamy et al. 2016). Larger arteries are generally associated with smooth muscle cells (SMCs) expressing $\alpha$-smooth muscle actin ( $\alpha$ SMA) and NG2. Within the metaphyseal region, capillary-associated perivascular cells express PDGFR $\beta$ and NG2, which are regulated by EC-secreted PDGF-B (Kusumbe et al. 2016). The same region also contains mesenchymal stem and progenitor cells expressing nestin and Scal (Itkin et al. 2016). It is currently not clear whether the metaphysis contains distinct subsets of mesenchymal cells or whether the expression of different markers reflects dynamic changes and plasticity within a larger mesenchymal population. In the diaphyseal region, sinusoidal type L vessels are also associated with at least two different kinds of perivascular support cells. The first are mesenchymal cells expressing leptin receptor (LEPR) and PDGFR $\alpha$ (Ding et al. 2012) and the second are CXCL12abundant reticular (CAR) cells (Sugiyama et al. 2006). Together with signals from the endothelium, vessel-associated mesenchymal cells help to maintain hematopoietic stem-cell populations (Sugiyama et al. 2006; Ding et al. 2012). As for the metaphysis, the exact relationship between each of the perivascular cell populations in the BM remains to be determined. Nevertheless, the different types of perivascular cells found associated with bone vasculature of distinct regions further highlight the differing functional roles that individual EC populations perform and their distinct local environments.

Interestingly, recent cell-tracing experiments have revealed that the identity of individual ECs is not fixed. Pulse-chase experiments using a fluorescent reporter expressed following tamoxifen-induced Apln-CreERT activation, which can be used to label angiogenic ECs in many different organs and settings (Liu et al. 2015), showed that type E ECs can give rise to type H ECs. In addition, both of these subtypes can differentiate into type $\mathrm{L}$ and AECs during postnatal development (Fig. 2) (Langen et al. 2017). It has not yet been shown that any of these specialized EC populations contributes to the venous vasculature within bone.

\section{ENDOTHELIAL CELL SIGNALING IN BONE PRODUCTION AND DESTRUCTION}

In addition to acting as a conduit for blood, the vasculature has also been shown to play an important and active role in tissue morphogenesis. Previous studies have demonstrated a role for ECs during the morphogenesis of a range of tissues, including the kidney (Majumdar and Drummond 1999), lung (Ding et al. 2011), and liver (Ding et al. 2010, 2014). ECs are also instructive in salivary gland patterning (Kwon et al. 2017) and are involved in pancreatic islet innervation (Reinert et al. 2014). ECs have also been identified to play a key role in bone formation and the role of individual EC subpopulations has been dissected using a range of genetic mouse models. In addition to the obvious coordinated timing of vascularization and bone formation in embryonic POCs (Maes et al. 2002; Dirckx et al. 2013; Maes 2013), it has also been shown that osteoblast precursors move into developing and fractured bones along with invading blood vessels, which were identified with the marker CD31 (Maes et al. 2010). More recently, type $H$ vessels were shown to play a key role in supporting osteogenesis. CD $31^{\text {hi }}$ Emcn $^{\text {hi }}$ ECs 
A

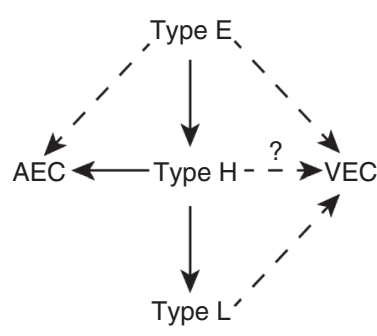

B

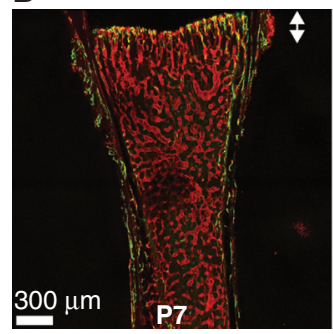

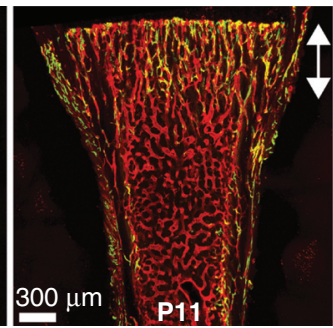

GFP (Apln-CreER) Emcn

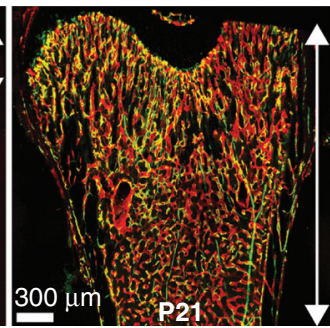

Figure 2. Relationship between specialized endothelial cell (EC) subpopulations. (A) Schematic diagram of potential EC fates. Type E ECs have the potential to become type H ECs, which in turn can give rise to type L ECs. Type H ECs have the potential to become arterial ECs (AECs), but it remains unclear whether type E ECs can do the same. The ability of type E, H, and L ECs to give rise to venous ECs (VECs) remains to be determined. Solid arrows indicate strong evidence; dashed arrows indicate possible connections. $(B)$ Arrows indicate expansion of $\mathrm{GFP}^{+}$vessels into the marrow cavity: green fluorescent protein (GFP) (Apln-CreER) (green) and endomucin (Emcn) (red). (Overview fluorescent images from Langen et al. 2017; reprinted, with permission, from Nature Publishing Group (c) 2017.)

were found to associate with Osterix-expressing osteoprogenitors and to express high levels of pro-osteogenic factors, such as bone morphogenetic proteins (BMPs), fibroblast growth factors (FGFs), and PDGFs, relative to the type L vasculature (Kusumbe et al. 2014). In vitro coculture experiments of freshly isolated bone ECs with mesenchymal cells directly confirmed that type $\mathrm{H}$ and type E ECs but not type $\mathrm{L}$ endothelium promote osteogenic differentiation processes independently of blood perfusion (Langen et al. 2017).

Similar to type H ECs, type E ECs are present for a distinct time period. They are particularly prevalent during late embryonic and early postnatal development, when extensive bone growth is occurring. Interestingly, Osterix-expressing osteoprogenitors were even more strongly associated with type $\mathrm{E}$ vessels than type $\mathrm{H}$ vessels at $\mathrm{P} 6$, because of high expression of factors such as BMPs (Langen et al. 2017). Both type $E$ and type $H$ vessels were shown to be highly dependent on cell-matrix signaling interactions to support their function in coupling angiogenesis and osteogenesis and share a requirement for integrin $\beta 1$. In its absence, bone vasculature was disorganized and showed changes in regional metabolic properties (Langen et al. 2017). It also resulted in a significant reduction in bone volume, number of trabeculae and trabecular thickness, as well as increased trabecular separation. This emphasizes the importance of these EC subpopulations and their interactions with the ECM to facilitate the coupling of angiogenesis and osteogenesis.

In the aging organism, osteoprogenitor number and bone mass decrease concomitantly with the number of type $\mathrm{H}$ vessels (Fig. 3) (Kusumbe et al. 2014). Pharmacologically induced maintenance of type $\mathrm{H}$ vessels in aging resulted in increased bone density compared to agematched controls (Kusumbe et al. 2014).

\section{VEGF IN THE COUPLING OF ANGIOGENESIS AND OSTEOGENESIS}

VEGF-A signaling plays a key role in regulating the angiogenic process and its coupling to osteogenesis. VEGFR2 is the main receptor for VEGF-A and is expressed on ECs where it can regulate their survival, migration, and proliferation (Chung and Ferrara 2011). VEGFR2 is also expressed by VEGF-producing cells of the osteoblast lineage and can regulate survival, migration, and differentiation through autocrine signaling processes (Duan et al. 2015; Hu and Olsen 2016). Loss of VEGF-A expression in osteoprogenitors was shown to inhibit their differentiation into mature bone-forming osteoblasts, resulting in reduced bone density (Liu et al. 
The Vasculature of the Skeletal System

A

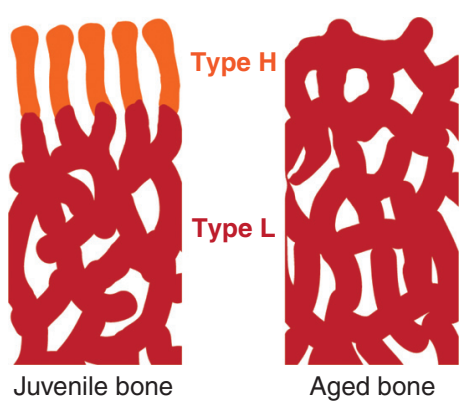

B Juvenile (4 weeks)

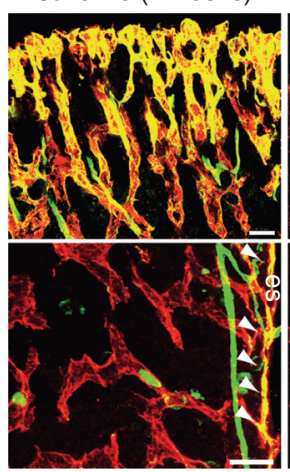

Adult (11 weeks)

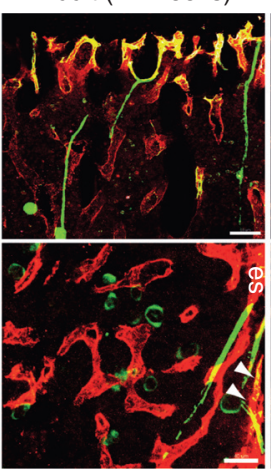

CD31 Emcn

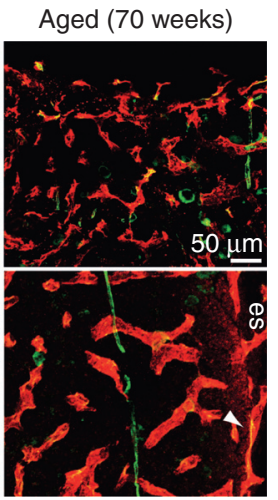

Figure 3. Changes in bone vasculature with age. (A) Schematic diagram of changes in endothelial cell (EC) subpopulations with age, where type $\mathrm{H}$ vessels (orange) are gradually replaced with type $\mathrm{L}$ (red) vessels over time. $(B)$ Overview fluorescent images of changes in metaphyseal (top panels) and diaphyseal (bottom panels) vessels with age. Note the age-dependent decline in type H ECs of the metaphysis and endosteum (es, arrowheads). (Image from Kusumbe et al. 2014; reprinted, with permission, from Nature Publishing Group (c) 2017.)

2012). Expression of VEGF-A by hypertrophic chondrocytes in the center of the developing bone drives the invasion of blood vessels and allows the formation of the POC. Administration of a soluble VEGF receptor (sFlt-1) reduced vascular invasion of the growth plate as well as trabecular bone formation and bone growth. It also resulted in an enlarged hypertrophic cartilage zone (Gerber et al. 1999).

There are three major isoforms of VEGFA expressed in mice: $\mathrm{VEGF}_{120}, \mathrm{VEGF}_{164}$ and $\mathrm{VEGF}_{188}$ (equivalent to human $\mathrm{VEGF}_{121}$, $\mathrm{VEGF}_{165}$, and $\mathrm{VEGF}_{189}$, respectively). $\mathrm{VEGF}_{120}$ is a freely diffusible isoform and its expression alone, in the absence of $\mathrm{VEGF}_{164}$ and $\mathrm{VEGF}_{188}$, is insufficient to support normal bone growth and formation (Maes et al. 2002). Mice expressing only the $\mathrm{VEGF}_{120}$ isoform $\left(V E G F^{120 / 120}\right.$ mice) display disturbed vascular patterning, reduced trabecular bone volume, and reduced bone growth (Maes et al. 2002). While expression of the $\mathrm{VEGF}_{188}$ isoform alone was sufficient for development of the metaphysis, it did not support normal development of the growth plate and SOCs, with reduced vascularization of the epiphysis and massive chondrocyte apoptosis observed in these regions (Maes et al. 2004). In contrast, expression of $\mathrm{VEGF}_{164}$ alone was sufficient for normal skeletal development to occur in the absence of the other isoforms (Maes et al. 2004).

Bone vasculature has been shown to directly inform the deposition pattern of the mineralized bone matrix by acting as a guiding template (Ben Shoham et al. 2016). During embryonic development, bone ECs were shown to be devoid of basement membrane. Instead, the vessels were coated with collagen type I secreted by osteoblasts, which could then undergo gradual mineralization. Defective vessel patterning induced through overexpression of $\mathrm{VEGF}_{165}$ also resulted in abnormal collagen I deposition in association with the vasculature (Ben Shoham et al. 2016).

\section{REGULATION OF ANGIOGENESIS AND OSTEOGENESIS BY HYPOXIA- INDUCIBLE FACTORS}

Hypoxia is a key driver during vascularization of the cartilage template required for bone formation. Hypoxia can be detected through transcriptional regulators known as hypoxia-inducible factors (HIFs). HIFs are heterodimers composed of one of three $\alpha$-subunits (HIF $1 \alpha$, HIF $2 \alpha$, or HIF $3 \alpha$ ) and a $\beta$-subunit (HIF1 $\beta$ ). At normal oxygen levels, HIF $1 \alpha$ and HIF $2 \alpha$ are ubiquitously expressed, but they are rapidly hy- 
droxylated at conserved proline residues by HIF prolyl-hydroxylases and thereby marked for proteasomal degradation through polyubiquitination by the E3 ligase von Hippel-Lindau (VHL) protein (Cockman et al. 2000). However, if oxygen levels fall below around 5\%, HIF1o and HIF $2 \alpha$ are no longer degraded and can form a complex with the HIF1 $\beta$ subunit. HIF signaling is important for chondrocyte survival. It allows them to adapt their cellular metabolism to the low oxygen environment (Rajpurohit et al. 1996). Loss of HIF1 $\alpha$ expression in chondrocytes leads to disorganized growth plates because of massive cell death of the inner hypoxic regions of the growth plates and defective chondrocyte proliferation and hypertrophy (Schipani et al. 2001). HIF1 $\alpha$ is involved in regulating the expression of VEGF-A in growth plate chondrocytes and osteoblasts (Wang et al. 2007). Loss of HIF1 $\alpha$ in osteoblasts reduces angiogenesis and osteogenesis in long bones, while overexpression of HIF $1 \alpha$ or loss of VHL in osteoblasts increases long bone angiogenesis and osteogenesis (Wang et al. 2007). Interestingly, mechanical stress in osteoblasts can also promote expression of HIF $1 \alpha$ though activation of Ras/ERK-mediated mitogen-activated protein kinase (MAPK) signaling (Wang et al. 2004). EC-specific activation of HIF signaling promotes the formation of type $\mathrm{H}$ capillaries and increases osteogenesis (Kusumbe et al. 2014). Together, these data demonstrate an important role for hypoxia signaling in the coupling of angiogenesis and osteogenesis.

\section{NOTCH SIGNALING IN THE COUPLING OF ANGIOGENESIS AND OSTEOGENESIS}

The Notch pathway regulates a variety of processes both during development and in the adult. In the vasculature, Notch signaling regulates blood vessel function and growth by modulating the response of ECs to growth factors such as VEGF-A (Harrington et al. 2008). In sprouting angiogenesis, new vessel sprouts are led by "tip cells," which are responsible for initiating and guiding new sprouts, and trailing behind them are the highly proliferative "stalk cells" (Gerhardt et al. 2003). Activation of VEGFR2 by VEGF-A in tip cells induces up- regulation of the Notch ligand Delta-like ligand 4 (Dll4). Dll4 activates Notch signaling in the neighboring stalk cells and thereby limits activities such as endothelial sprouting and proliferation. Accordingly, Notch signaling has been observed to inhibit blood vessel growth in the mouse retina (Hellstrom et al. 2007), zebrafish embryos (Siekmann and Lawson 2007), and tumors (Ridgway et al. 2006). Surprisingly though, active Notch signaling in the bone endothelium promotes angiogenesis rather than inhibiting it (Ramasamy et al. 2014). EC-specific inactivation of Notch signaling through inactivation of Dll4 or $R b p j$, which encodes RBP-J $\kappa$, an essential mediator of Notch-induced gene transcription, leads to a reduction in angiogenesis, loss of type $\mathrm{H}$ vasculature, and reduced bone formation (Ramasamy et al. 2014). While the reasons for the organ-specific effects of Notch on angiogenic growth remain incompletely understood, Hes5, one of the transcriptional regulators acting downstream of active Notch, was shown to be differentially expressed in bone and lung ECs (Ramasamy et al. 2014). In addition, endothelial Notch signaling leads to the expression of Noggin, a secreted BMP antagonist and important regulator of chondrocyte and osteoblast behavior (Brunet et al. 1998; Tylzanowski et al. 2006). Hypertrophic chondrocytes are an essential source of VEGF-A (Gerber et al. 1999), which, in turn, is well known to induce Dll4 expression and thereby Notch activity in ECs (Hellstrom et al. 2007; Benedito et al. 2009). Accordingly, expression of VEGF-A in the adolescent growth plate was strongly diminished in EC-specific Rbpj mutant mice (Ramasamy et al. 2014). Together, these data show that the behavior of ECs and growth plate chondrocytes is tightly coupled through secreted signals generating a positive Notch-dependent feedback loop controlling angiogenesis and osteogenesis (Fig. 4).

Endothelial-specific $R b p j$ mutants also showed a down-regulation of flow-modulated genes Klf2, Nos3, and Pecam1, suggesting that flow may play a role in regulating angiogenesis and osteogenesis through regulation of Notch signaling (Ramasamy et al. 2016). Pharmacological reduction of blood flow led to a reduction in endothelial Dll4 expression as well as loss of 
A

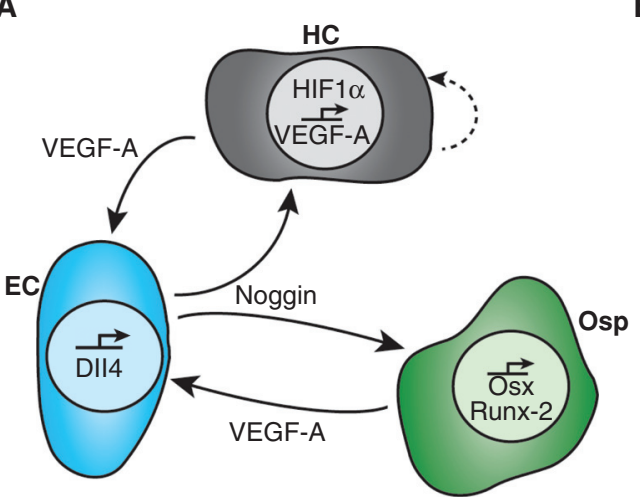

B

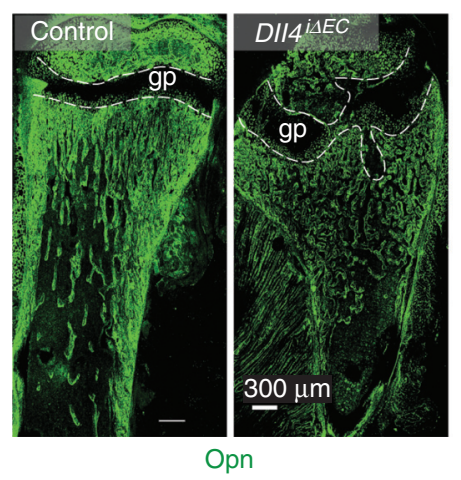

C

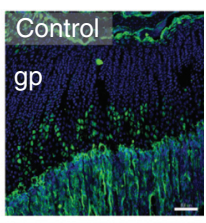

$R b p j i \triangle E C$

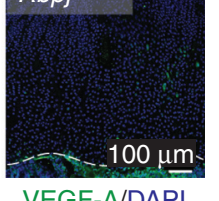

Figure 4. Notch signaling in the coupling of angiogenesis and osteogenesis. (A) Schematic diagram of the interaction between hypertrophic chondrocytes (HCs, gray), endothelial cells (ECs, blue), and osteoprogenitors (Osp, green). The hypoxic environment surrounding the HCs leads to accumulation of hypoxia-inducible factor (HIF) $1 \alpha$ and subsequent expression of vascular endothelial growth factor A (VEGF-A). The VEGF-A acts as an autocrine signal for the $\mathrm{HC}$ as well as a paracrine signal for the neighboring EC. This induces Delta-like ligand 4 (Dll4) expression in the ECs and activates Notch signaling. Noggin is released and acts on both the HC and the Osp. Noggin promotes the generation of osteoblast lineage cells by inducing expression of Osx and Runx-2, thereby facilitating bone formation. (B) Osteopontin (Opn, green) immunostaining in 4-week-old mice lacking endothelial Dll4 (Dll4 ${ }^{i \triangle E C}$ ), which display malformed bone and growth plate (gp, dashed lines). (C) Strongly reduced VEGF-A immunostaining (green) in the gp of P28 mice lacking endothelial RBP-JK $\left(R b p j^{i \Delta E C}\right.$ ), a transcription factor mediating Notch-dependent gene expression. (Fluorescent images from Ramasamy et al. 2014; reprinted, with permission, from Nature Publishing Group ( 2014.)

mineralized bone. Furthermore, EC-specific reactivation of Notch signaling in aged mice was able to significantly increase type $\mathrm{H}$ vasculature and promote angiogenesis and osteogenesis (Ramasamy et al. 2016).

\section{FIBROBLAST GROWTH FACTOR SIGNALING IN ANGIOGENESIS AND OSTEOGENESIS}

The FGF family is made up of 18 secreted factors and four receptor tyrosine kinases (FGFRs). FGFs are capable of regulating expression of VEGF-A and VEGFR2 (Behr et al. 2010; Murakami et al. 2011). FGFs are secreted by chondrocytes and osteogenic cells, while blood vessels of the bone have been shown to express FGFR1 and FGFR2. As such, FGF signaling may regulate bone angiogenesis and osteogenesis upstream of VEGF signaling. Mice with EC-specific loss of FGFR1 and FGFR2 show abnormalities in bone vasculature, with decreased arterial density and diameter as well as increased sinusoidal diameter (Itkin et al. 2016). FGF2-deficient mice show reduced trabecular bone volume, mineral apposition, and bone-formation rates (Montero et al. 2000). Overall, these studies indicate that FGF signaling is required for normal bone production and vascularization.

\section{DYSREGULATION OF BONE VASCULATURE IN AGING AND OSTEOPOROSIS}

The vasculature of the skeletal system is essential for the formation of new bone in development; however, vasculature can also play a role in a pathological context. Bone remodeling continues over the course of a lifetime and involves the repair of microfractures and the constant replacement of older bone. This renewal involves a coordinated response from osteoclasts, which dissolve bone, and osteoblasts, which produce new bone. If the balance between these two processes becomes dysregulated or dysfunctional, it can lead to a range of pathological conditions. The role of bone vasculature in a range of bone pathologies is only beginning to emerge. As part of the normal aging process, bone density gradually decreases (Demontiero et al. 2012). How- 
ever, in some people, the rate of bone density loss is dramatically increased and can lead to a condition known as osteoporosis. Osteoporosis is an age-related disease that occurs in both men and women, but is particularly prevalent in postmenopausal women and causes increased bone fragility and susceptibility to fractures.

Developmental studies demonstrate a strong interdependency between osteogenesis and angiogenesis, and there are data suggesting that the same is also true in postmenopausal osteoporosis. The primary cause of postmenopausal osteoporosis is estrogen deficiency (Riggs et al. 1998). As such, ovariectomized rodents can recapitulate many aspects of postmenopausal osteoporosis and can be used to further understand the disease. Postmenopausal osteoporosis is associated with significantly decreased bone density, a reduction in sinusoidal and arterial capillaries, and reduced bone perfusion. Loss of these vessels in postmenopausal osteoporosis has been reported to cause reduced bone perfusion (Shih et al. 2004). Inactivation of VHL in osteoblasts of ovariectomized mice increases HIF $1 \alpha$, HIF $2 \alpha$, and VEGF-A levels compared to wild-type ovariectomized mice. This protects the ovariectomized mice from loss of bone vasculature, bone density, and mechanical strength (Zhao et al. 2012). Prevention of bone blood vessel loss through HIF signaling may not only improve bone density through improved perfusion in postmenopausal osteoporosis, but may also influence the endothelial subpopulations present.

In normal aging, type $\mathrm{H}$ vessels are gradually lost (Fig. 3), coinciding with a reduction in osteogenesis, bone quality, and fracture healing (Smith et al. 1975; Kusumbe et al. 2014; Xie et al. 2014). In mice, inactivation of VHL in ECs leads to an increase in type $\mathrm{H}$ vasculature as well as increased trabecular bone formation and increased osteoprogenitors (Kusumbe et al. 2014). Treatment of aged mice with deferoxamine mesylate (DFM), which enhances HIF1 $\alpha$ stability and activity, also resulted in increased type $\mathrm{H}$ vasculature and increased bone mass (Kusumbe et al. 2014). In contrast, ovariectomized mice show a decrease in type $\mathrm{H}$ vessels and associated osteoprogenitors (Xie et al. 2014). Human pa- tients suffering osteoporosis or osteopenia, also show a reduction in type $\mathrm{H}$ vasculature when compared to age-matched controls (Wang et al. 2017). Preosteoclasts may also play a role in the pathogenesis of postmenopausal osteoporosis, as secretion of PDGF-B by preosteoclasts is able to increase the number of type $\mathrm{H}$ vessels in ovariectomized mice and promote osteogenesis (Xie et al. 2014). Together, these data strongly indicate a key role for bone vasculature, particularly type $\mathrm{H}$ vasculature, in the progression and severity of postmenopausal osteoporosis, and the possibility that increasing or maintaining type $\mathrm{H}$ vasculature in an osteoporosis context may improve bone density in human patients.

\section{FRACTURE REPAIR}

Bone fractures are the most common large-organ traumatic injury in humans (Einhorn and Gerstenfeld 2015), but bone is one of the few tissues in adult humans that can fully regenerate. Bone fracture healing is a multistep process. It commences with the formation of a hematoma around the fractured region of the bone, which attracts cytokine-secreting inflammatory cells that help to form a fibrinous clot (Gerstenfeld et al. 2003). Similar to development, bone formation and repair following injury can proceed via either endochondral ossification or intramembranous ossification. Whereas unstable, hypoxic fractures generally repair via endochondral ossification, stable bone fractures generally have sufficient access to oxygen, nutrients, and trophic factors to allow direct differentiation of mesenchymal cells to osteoblasts, as occurs in intramembranous ossification (Dirckx et al. 2013). In the case of endochondral ossification, high levels of VEGF-A within the hematoma promote vascular invasion, allowing the formation of a soft callus. The soft callus contains osteoblasts to facilitate bone formation, as well as chondrocytes that contribute to the formation of new cartilage. This then matures into a hard callus before being remodeled into mature bone. Blood supply is essential for callus formation during fracture healing and repair (Trueta 1974), with around $10 \%$ of all bone fracture patients showing compromised fracture healing 
because of lack of blood supply after injury (Bishop et al. 2012; Gomez-Barrena et al. 2015). If blood flow is impaired, it can delay bone healing and repair (Wan et al. 2008), and it has been shown that pharmacological inhibition of angiogenesis prevents callus formation in fracture healing and causes formation of fibrous tissue (Hausman et al. 2001). Blood vessels are not only required to supply oxygen and nutrients during bone repair, but vessel invasion is also essential to facilitate the entry of osteoblast precursors into the fracture callus (Maes et al. 2010).

In addition to VEGF-A, numerous other factors and cytokines contribute to bone vascularization and repair following fracture, including placental growth factor (PIGF), FGF, PDGF, insulin-like growth factor (IGF), and BMP. PIGF, a member of the VEGF family, is required for normal fracture healing, and mice lacking Plgf show impaired fracture wound vascularization, osteogenesis, and cartilage turnover (Maes et al. 2006). As is the case during embryonic development of bones, FGF signaling is also required during bone repair. Mice with heterozygous loss of FGF9 showed reduced neovascularization and cortical repair, in part because of reduced VEGF-A (Behr et al. 2010). Combined administration of FGF9 and VEGF-A was able to rescue this defect. Furthermore, exogenous FGF9 administration in wild-type mice was able to accelerate bone repair (Behr et al 2010). In contrast, FGF18 has been shown to be important for cortical bone healing independent of angiogenesis (Behr et al. 2011).

\section{AVASCULAR NECROSIS}

Blood vessels are essential for the continued maintenance and survival of bones. Loss of bone vasculature can result in diseases such as avascular necrosis (also termed osteonecrosis). Avascular necrosis can occur in any bone of the body; however, it most commonly affects the femoral head (Agarwala et al. 2002). Avascular necrosis of the femoral head (ANFH) involves the disruption of blood flow, which can be caused by damage to the EC layer of vessels and blood clot formation. The subsequent ische- mia can result in necrotic death of osteocytes and collapse of the articular surface and ultimately even the onset of osteoarthritis (Filipowska et al. 2017). There are numerous factors that can cause or increase susceptibility to ANFH, including traumatic injury to the femur, hip dislocation, steroid use (Chan and Mok 2012), and excessive alcohol consumption (Jacobs 1992). While the pathogenesis is not completely understood, previous work showed that patients with nontraumatic ANFH had lower numbers of endothelial progenitor cells and that these cells were less migratory and more senescent than in healthy subjects, leading to poor angiogenic capability (Feng et al. 2010). These data emphasize the importance of sustained perfusion of bone vessels for continued bone functionality.

\section{CONCLUDING REMARKS}

It is becoming increasingly apparent that the vasculature of the bone is not only essential for oxygen and nutrient supply, but also plays a key role in the formation and maintenance of bone and BM. Recent work identified distinct, specialized blood vessel types that perform unique functions during bone growth, maintenance, and repair. The coupling of osteogenesis and angiogenesis is directly influenced by the cross talk between specific EC populations, osteoblasts, and chondrocytes. This cross talk is dependent upon multiple signaling pathways, including VEGF, HIF, Notch, BMP, and FGF signaling.

In aging, the vascular composition changes such that the osteogenesis-promoting type $\mathrm{H}$ vasculature is gradually replaced with type $\mathrm{L}$ vessels, with concomitant bone density loss. Bone vasculature plays a role in a range of bone pathologies. While in many cases the relationship between the bone vasculature and disease pathogenesis is not completely understood, there is evidence that ECs of the bones are directly involved and can influence outcomes in cases such as fracture repair and avascular necrosis. A greater understanding of the role of bone vasculature in both development and disease may lead to new therapeutic approaches in a range of bone conditions. 
E.C. Watson and R.H. Adams

\section{ACKNOWLEDGMENTS}

We thank the Max Planck Society, the University of Münster, the German Research Foundation (DFG) Clusters of Excellence "Cells in Motion," and the European Research Council (AdG 339409 AngioBone) for support and funding.

\section{REFERENCES}

Agarwala S, Sule A, Pai BU, Joshi VR. 2002. Alendronate in the treatment of avascular necrosis of the hip. Rheuma tology (Oxford) 41: 346-347.

Asada N, Takeishi S, Frenette PS. 2017. Complexity of bone marrow hematopoietic stem cell niche. Int J Hematol 106: 45-54.

Behr B, Leucht P, Longaker MT, Quarto N. 2010. Fgf-9 is required for angiogenesis and osteogenesis in long bone repair. Proc Natl Acad Sci 107: 11853-11858.

Behr B, Sorkin M, Manu A, Lehnhardt M, Longaker MT, Quarto N. 2011. Fgf-18 is required for osteogenesis but not angiogenesis during long bone repair. Tissue Eng Part A 17: 2061-2069.

Benedito R, Roca C, Sorensen I, Adams S, Gossler A, Fruttiger M, Adams RH. 2009. The notch ligands Dll4 and Jagged1 have opposing effects on angiogenesis. Cell 137: 1124-1135.

Bennett HS, Luft JH, Hampton JC. 1959. Morphological classifications of vertebrate blood capillaries. Am J Physiol 196: 381-390.

Ben Shoham A, Rot C, Stern T, Krief S, Akiva A, Dadosh T, Sabany H, Lu Y, Kadler KE, Zelzer E. 2016. Deposition of collagen type I onto skeletal endothelium reveals a new role for blood vessels in regulating bone morphology. Development 143: 3933-3943.

Bishop JA, Palanca AA, Bellino MJ, Lowenberg DW. 2012. Assessment of compromised fracture healing. J Am Acad Orthop Surg 20: 273-282.

Bixel MG, Kusumbe AP, Ramasamy SK, Sivaraj KK, Butz S, Vestweber D, Adams RH. 2017. Flow dynamics and HSPC homing in bone marrow microvessels. Cell Rep 18: $1804-1816$.

Brachtendorf G, Kuhn A, Samulowitz U, Knorr R, Gustafsson E, Potocnik AJ, Fassler R, Vestweber D. 2001. Early expression of endomucin on endothelium of the mouse embryo and on putative hematopoietic clusters in the dorsal aorta. Dev Dyn 222: 410-419.

Brunet LJ, McMahon JA, McMahon AP, Harland RM. 1998. Noggin, cartilage morphogenesis, and joint formation in the mammalian skeleton. Science 280: 14551457.

Chan KL, Mok CC. 2012. Glucocorticoid-induced avascular bone necrosis: Diagnosis and management. Open Orthop J 6: 449-457.

Chung AS, Ferrara N. 2011. Developmental and pathological angiogenesis. Annu Rev Cell Dev Biol 27: 563-584.

Cockman ME, Masson N, Mole DR, Jaakkola P, Chang GW, Clifford SC, Maher ER, Pugh CW, Ratcliffe PJ, Maxwell PH. 2000. Hypoxia inducible factor- $\alpha$ binding and ubiq- uitylation by the von Hippel-Lindau tumor suppressor protein. J Biol Chem 275: 25733-25741.

Coultas L, Chawengsaksophak K, Rossant J. 2005. Endothelial cells and VEGF in vascular development. Nature 438: 937-945.

Demontiero O, Vidal C, Duque G. 2012. Aging and bone loss: New insights for the clinician. Ther Adv Musculoskelet Dis 4: 61-76.

Ding L, Morrison SJ. 2013. Haematopoietic stem cells and early lymphoid progenitors occupy distinct bone marrow niches. Nature 495: 231-235.

Ding BS, Nolan DJ, Butler JM, James D, Babazadeh AO, Rosenwaks Z, Mittal V, Kobayashi H, Shido K, Lyden D, et al. 2010. Inductive angiocrine signals from sinusoidal endothelium are required for liver regeneration. Nature 468: 310-315.

Ding BS, Nolan DJ, Guo P, Babazadeh AO, Cao Z, Rosenwaks Z, Crystal RG, Simons M, Sato TN, Worgall S, et al. 2011. Endothelial-derived angiocrine signals induce and sustain regenerative lung alveolarization. Cell 147: 539-553.

Ding L, Saunders TL, Enikolopov G, Morrison SJ. 2012. Endothelial and perivascular cells maintain haematopoietic stem cells. Nature 481: 457-462.

Ding BS, Cao Z, Lis R, Nolan DJ, Guo P, Simons M, Penfold ME, Shido K, Rabbany SY, Rafii S. 2014. Divergent angiocrine signals from vascular niche balance liver regeneration and fibrosis. Nature 505: 97-102.

Dirckx N, Van Hul M, Maes C. 2013. Osteoblast recruitment to sites of bone formation in skeletal development, homeostasis, and regeneration. Birth Defects Res C Embryo Today 99: 170-191.

Duan X, Murata Y, Liu Y, Nicolae C, Olsen BR, Berendsen AD. 2015. Vegfa regulates perichondrial vascularity and osteoblast differentiation in bone development. Development 142: 1984-1991.

Einhorn TA, Gerstenfeld LC. 2015. Fracture healing: Mechanisms and interventions. Nat Rev Rheumatol 11: 45-54.

Feng Y, Yang SH, Xiao BJ, Xu WH, Ye SN, Xia T, Zheng D, Liu XZ, Liao YF. 2010. Decreased in the number and function of circulation endothelial progenitor cells in patients with avascular necrosis of the femoral head. Bone 46: 32-40.

Filipowska J, Tomaszewski KA, Niedzwiedzki L, Walocha JA, Niedzwiedzki T. 2017. The role of vasculature in bone development, regeneration and proper systemic functioning. Angiogenesis 20: 291-302.

Fish JE, Wythe JD. 2015. The molecular regulation of arteriovenous specification and maintenance. Dev Dyn 244: 391-409.

Franco CA, Jones ML, Bernabeu MO, Vion AC, Barbacena P, Fan J, Mathivet T, Fonseca CG, Ragab A, Yamaguchi TP, et al. 2016. Non-canonical Wnt signalling modulates the endothelial shear stress flow sensor in vascular remodelling. eLife 5: e07727.

Galie PA, Nguyen DH, Choi CK, Cohen DM, Janmey PA, Chen CS. 2014. Fluid shear stress threshold regulates angiogenic sprouting. Proc Natl Acad Sci 111: 7968-7973.

Gerber HP, Vu TH, Ryan AM, Kowalski J, Werb Z, Ferrara N. 1999. VEGF couples hypertrophic cartilage remodel- 
ing, ossification and angiogenesis during endochondral bone formation. Nat Med 5: 623-628.

Gerhardt H, Golding M, Fruttiger M, Ruhrberg C, Lundkvist A, Abramsson A, Jeltsch M, Mitchell C, Alitalo K, Shima $\mathrm{D}$, et al. 2003. VEGF guides angiogenic sprouting utilizing endothelial tip cell filopodia. J Cell Biol 161: 1163-1177.

Gerstenfeld LC, Cullinane DM, Barnes GL, Graves DT, Einhorn TA. 2003. Fracture healing as a post-natal developmental process: Molecular, spatial, and temporal aspects of its regulation. J Cell Biochem 88: 873-884.

Gomez-Barrena E, Rosset P, Lozano D, Stanovici J, Ermthaller C, Gerbhard F. 2015. Bone fracture healing: Cell therapy in delayed unions and nonunions. Bone 70: 93-101.

Harrington LS, Sainson RC, Williams CK, Taylor JM, Shi W, Li JL, Harris AL. 2008. Regulation of multiple angiogenic pathways by Dll4 and Notch in human umbilical vein endothelial cells. Microvasc Res 75: 144-154.

Hausman MR, Schaffler MB, Majeska RJ. 2001. Prevention of fracture healing in rats by an inhibitor of angiogenesis. Bone 29: 560-564.

Hellstrom M, Phng LK, Hofmann JJ, Wallgard E, Coultas L, Lindblom P, Alva J, Nilsson AK, Karlsson L, Gaiano N, et al. 2007. Dll4 signalling through Notch1 regulates formation of tip cells during angiogenesis. Nature 445: 776 780 .

Hu K, Olsen BR. 2016. Osteoblast-derived VEGF regulates osteoblast differentiation and bone formation during bone repair. J Clin Invest 126: 509-526.

Hu J, Srivastava K, Wieland M, Runge A, Mogler C, Besemfelder E, Terhardt D, Vogel MJ, Cao L, Korn C, et al. 2014. Endothelial cell-derived angiopoietin-2 controls liver regeneration as a spatiotemporal rheostat. Science 343: 416419.

Itkin T, Gur-Cohen S, Spencer JA, Schajnovitz A, Ramasamy SK, Kusumbe AP, Ledergor G, Jung Y, Milo I, Poulos MG et al. 2016. Distinct bone marrow blood vessels differentially regulate haematopoiesis. Nature 532: 323-328.

Jacobs B. 1992. Alcoholism-induced bone necrosis. NY State J Med 92: 334-338.

Kamba T, Tam BY, Hashizume H, Haskell A, Sennino B, Mancuso MR, Norberg SM, O’Brien SM, Davis RB, Gowen LC, et al. 2006. VEGF-dependent plasticity of fenestrated capillaries in the normal adult microvasculature. Am J Physiol Heart Circ Physiol 290: H560-H576.

Kunisaki Y, Bruns I, Scheiermann C, Ahmed J, Pinho S, Zhang D, Mizoguchi T, Wei Q, Lucas D, Ito K, et al. 2013. Arteriolar niches maintain haematopoietic stem cell quiescence. Nature 502: 637-643.

Kusumbe AP, Ramasamy SK, Adams RH. 2014. Coupling of angiogenesis and osteogenesis by a specific vessel subtype in bone. Nature 507: 323-328.

Kusumbe AP, Ramasamy SK, Itkin T, Mae MA, Langen UH, Betsholtz C, Lapidot T, Adams RH. 2016. Age-dependent modulation of vascular niches for haematopoietic stem cells. Nature 532: $380-384$.

Kwon HR, Nelson DA, DeSantis KA, Morrissey JM, Larsen M. 2017. Endothelial cell regulation of salivary gland epithelial patterning. Development 144: 211-220.

Langen UH, Pitulescu ME, Kim JM, Enriquez-Gasca R, Sivaraj KK, Kusumbe AP, Singh A, Di Russo J, Bixel MG Zhou B, et al. 2017. Cell-matrix signals specify bone en- dothelial cells during developmental osteogenesis. Nat Cell Biol 19: 189-201.

Liu Y, Berendsen AD, Jia S, Lotinun S, Baron R, Ferrara N, Olsen BR. 2012. Intracellular VEGF regulates the balance between osteoblast and adipocyte differentiation. $J$ Clin Invest 122: 3101-3113.

Liu Q, Hu T, He L, Huang X, Tian X, Zhang H, He L, Pu W, Zhang L, Sun H, et al. 2015. Genetic targeting of sprouting angiogenesis using Apln-CreER. Nat Commun 6: 6020.

Maes C. 2013. Role and regulation of vascularization processes in endochondral bones. Calcif Tissue Int 92: 307323.

Maes C, Carmeliet P, Moermans K, Stockmans I, Smets N, Collen D, Bouillon R, Carmeliet G. 2002. Impaired angiogenesis and endochondral bone formation in mice lacking the vascular endothelial growth factor isoforms VEGF164 and VEGF188. Mech Dev 111: 61-73.

Maes C, Stockmans I, Moermans K, Van Looveren R, Smets N, Carmeliet P, Bouillon R, Carmeliet G. 2004. Soluble VEGF isoforms are essential for establishing epiphyseal vascularization and regulating chondrocyte development and survival. J Clin Invest 113: 188-199.

Maes C, Coenegrachts L, Stockmans I, Daci E, Luttun A, Petryk A, Gopalakrishnan R, Moermans K, Smets N, Verfaillie CM, et al. 2006. Placental growth factor mediates mesenchymal cell development, cartilage turnover, and bone remodeling during fracture repair. J Clin Invest 116: $1230-1242$.

Maes C, Kobayashi T, Selig MK, Torrekens S, Roth SI, Mackem S, Carmeliet G, Kronenberg HM. 2010. Osteoblast precursors, but not mature osteoblasts, move into developing and fractured bones along with invading blood vessels. Dev Cell 19: 329-344.

Majumdar A, Drummond IA. 1999. Podocyte differentiation in the absence of endothelial cells as revealed in the zebrafish avascular mutant, cloche. Dev Genet 24: 220 229.

Mendez-Ferrer S, Michurina TV, Ferraro F, Mazloom AR, Macarthur BD, Lira SA, Scadden DT, Ma'ayan A, Enikolopov GN, Frenette PS. 2010. Mesenchymal and haematopoietic stem cells form a unique bone marrow niche. Nature 466: 829-834.

Mendez-Ferrer S, Scadden DT, Sanchez-Aguilera A. 2015. Bone marrow stem cells: Current and emerging concepts. Ann NY Acad Sci 1335: 32-44.

Montero A, Okada Y, Tomita M, Ito M, Tsurukami H, Nakamura T, Doetschman T, Coffin JD, Hurley MM. 2000. Disruption of the fibroblast growth factor-2 gene results in decreased bone mass and bone formation. J Clin Invest 105: 1085-1093.

Morrison SJ, Scadden DT. 2014. The bone marrow niche for haematopoietic stem cells. Nature 505: 327-334.

Murakami M, Nguyen LT, Hatanaka K, Schachterle W, Chen PY, Zhuang ZW, Black BL, Simons M. 2011. FGF-dependent regulation of VEGF receptor 2 expression in mice. $J$ Clin Invest 121: 2668-2678.

Pannarale L, Morini S, D’Ubaldo E, Gaudio E, Marinozzi G. 1997. SEM corrosion-casts study of the microcirculation of the flat bones in the rat. Anat Rec 247: 462-471.

Percival CJ, Richtsmeier JT. 2013. Angiogenesis and intramembranous osteogenesis. Dev Dyn 242: 909-922. 
E.C. Watson and R.H. Adams

Potente M, Makinen T. 2017. Vascular heterogeneity and specialization in development and disease. Nat Rev Mol Cell Biol 8: 477-494.

Potente M, Gerhardt H, Carmeliet P. 2011. Basic and therapeutic aspects of angiogenesis. Cell 146: 873-887.

Rajpurohit R, Koch CJ, Tao Z, Teixeira CM, Shapiro IM. 1996. Adaptation of chondrocytes to low oxygen tension: Relationship between hypoxia and cellular metabolism. $J$ Cell Physiol 168: 424-432.

Ramasamy SK, Kusumbe AP, Wang L, Adams RH. 2014 Endothelial Notch activity promotes angiogenesis and osteogenesis in bone. Nature 507: 376-380.

Ramasamy SK, Kusumbe AP, Adams RH. 2015. Regulation of tissue morphogenesis by endothelial cell-derived signals. Trends Cell Biol 25: 148-157.

Ramasamy SK, Kusumbe AP, Schiller M, Zeuschner D, Bixel MG, Milia C, Gamrekelashvili J, Limbourg A, Medvinsky A, Santoro MM, et al. 2016. Blood flow controls bone vascular function and osteogenesis. Nat Commun 7: 13601.

Reinert RB, Cai Q, Hong JY, Plank JL, Aamodt K, Prasad N, Aramandla R, Dai C, Levy SE, Pozzi A, et al. 2014. Vascular endothelial growth factor coordinates islet innervation via vascular scaffolding. Development 141: 14801491.

Ridgway J, Zhang G, Wu Y, Stawicki S, Liang WC, Chanthery Y, Kowalski J, Watts RJ, Callahan C, Kasman I, et al. 2006. Inhibition of Dll4 signalling inhibits tumour growth by deregulating angiogenesis. Nature 444: 1083-1087.

Riggs BL, Khosla S, Melton LJ III. 1998. A unitary model for involutional osteoporosis: Estrogen deficiency causes both type I and type II osteoporosis in postmenopausal women and contributes to bone loss in aging men. J Bone Miner Res 13: 763-773.

Risau W, Flamme I. 1995. Vasculogenesis. Annu Rev Cell Dev Biol 11: 73-91.

Schipani E, Ryan HE, Didrickson S, Kobayashi T, Knight M, Johnson RS. 2001. Hypoxia in cartilage: HIF-1 $\alpha$ is essential for chondrocyte growth arrest and survival. Genes Dev 15: 2865-2876.

Shih TT, Liu HC, Chang CJ, Wei SY, Shen LC, Yang PC. 2004. Correlation of MR lumbar spine bone marrow perfusion with bone mineral density in female subjects. $R a$ diology 233: 121-128.

Siekmann AF, Lawson ND. 2007. Notch signalling limits angiogenic cell behaviour in developing zebrafish arteries. Nature 445: 781-784.

Smith DM, Khairi MR, Johnston CC Jr. 1975. The loss of bone mineral with aging and its relationship to risk of fracture. J Clin Invest 56: 311-318.
Spencer JA, Ferraro F, Roussakis E, Klein A, Wu J, Runnels JM, Zaher W, Mortensen LJ, Alt C, Turcotte R, et al. 2014. Direct measurement of local oxygen concentration in the bone marrow of live animals. Nature 508: 269-273.

Sugiyama T, Kohara H, Noda M, Nagasawa T. 2006. Maintenance of the hematopoietic stem cell pool by CXCL12CXCR4 chemokine signaling in bone marrow stromal cell niches. Immunity 25: 977-988.

Sumpio BE, Riley JT, Dardik A. 2002. Cells in focus: Endothelial cell. Int J Biochem Cell Biol 34: 1508-1512.

Tomlinson RE, Silva MJ. 2013. Skeletal blood flow in bone repair and maintenance. Bone Res 1: 311-322.

Trueta J. 1974. Blood supply and the rate of healing of tibial fractures. Clin Orthop Relat Res 105: 11-26.

Trueta J, Morgan JD. 1960. The vascular contribution to osteogenesis. I: Studies by the injection method. J Bone Joint Surg Br 42-B: 97-109.

Tylzanowski P, Mebis L, Luyten FP. 2006. The Noggin null mouse phenotype is strain dependent and haploinsufficiency leads to skeletal defects. Dev Dyn 235: 1599-1607.

Wan C, Gilbert SR, Wang Y, Cao X, Shen X, Ramaswamy G, Jacobsen KA, Alaql ZS, Eberhardt AW, Gerstenfeld LC, et al. 2008. Activation of the hypoxia-inducible factor- $1 \alpha$ pathway accelerates bone regeneration. Proc Natl Acad Sci 105: 686-691.

Wang FS, Wang CJ, Chen YJ, Chang PR, Huang YT, Sun YC, Huang HC, Yang YJ, Yang KD. 2004. Ras induction of superoxide activates ERK-dependent angiogenic transcription factor HIF-1 $\alpha$ and VEGF-A expression in shock wave-stimulated osteoblasts. J Biol Chem 279: 1033110337.

Wang Y, Wan C, Deng L, Liu X, Cao X, Gilbert SR, Bouxsein ML, Faugere MC, Guldberg RE, Gerstenfeld LC, et al. 2007. The hypoxia-inducible factor $\alpha$ pathway couples angiogenesis to osteogenesis during skeletal development. J Clin Invest 117: 1616-1626.

Wang L, Zhou F, Zhang P, Wang H, Qu Z, Jia P, Yao Z, Shen G, Li G, Zhao G, et al. 2017. Human type H vessels are a sensitive biomarker of bone mass. Cell Death Dis 8: e2760.

Xie H, Cui Z, Wang L, Xia Z, Hu Y, Xian L, Li C, Xie L, Crane J, Wan M, et al. 2014. PDGF-BB secreted by preosteoclasts induces angiogenesis during coupling with osteogenesis. Nat Med 20: 1270-1278.

Zamboni L, Pease DC. 1961. The vascular bed of red bone marrow. J Ultrastruct Res 5: 65-85.

Zhao Q, Shen X, Zhang W, Zhu G, Qi J, Deng L. 2012. Mice with increased angiogenesis and osteogenesis due to conditional activation of HIF pathway in osteoblasts are protected from ovariectomy induced bone loss. Bone 50: 763-770 


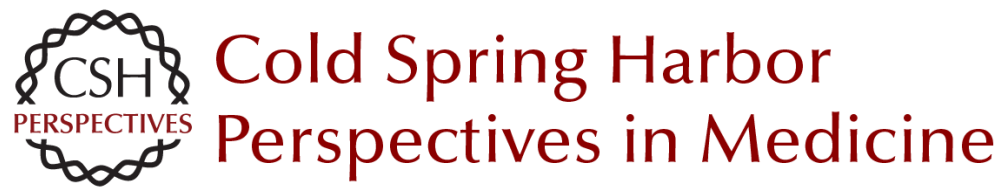

\section{Biology of Bone: The Vasculature of the Skeletal System}

Emma C. Watson and Ralf H. Adams

Cold Spring Harb Perspect Med 2018; doi: 10.1101/cshperspect.a031559 originally published online September 11, 2017

\section{Subject Collection Bone: A Regulator of Physiology}

\section{Mechanism of Bone Mineralization} Monzur Murshed

Neural Regulation of Bone and Bone Marrow Maria Maryanovich, Shoichiro Takeishi and Paul S. Frenette

Regulation of Bone Remodeling by Parathyroid Hormone Marc N. Wein and Henry M. Kronenberg

The Bone Marrow Microenvironment in Health and Myeloid Malignancy

Marta Galán-Díez, Álvaro Cuesta-Domínguez and Stavroula Kousteni

The Biology of Bone Metastasis

Mark Esposito, Theresa Guise and Yibin Kang

\section{Bone Remodeling and the Microbiome} Roberto Pacifici
Osteoimmunology
Kazuo Okamoto and Hiroshi Takayanagi

Multiple Myeloma and Bone: The Fatal Interaction Silvia Marino and G. David Roodman

Biology of Bone: The Vasculature of the Skeletal System

Emma C. Watson and Ralf H. Adams

Regulation of Energy Metabolism by

Bone-Derived Hormones

Paula Mera, Mathieu Ferron and Ioanna Mosialou

\section{Biology of Fibroblast Growth Factor 23: From \\ Physiology to Pathology \\ Marie Courbebaisse and Beate Lanske}

Regulation of Bone Metabolism by Sex Steroids Sundeep Khosla and David G. Monroe

For additional articles in this collection, see http://perspectivesinmedicine.cshlp.org/cgi/collection/ 